\title{
Genome Characterization of the First Outbreak of COVID-19 Delta Variant B.1.617.2 - Guangzhou City, Guangdong Province, China, May 2021
}

\author{
Zhencui $\mathrm{Li}^{1,2,2 \times}$; Kai Nie ${ }^{3,8 x}$; Kuibiao $\mathrm{Li}^{4} ;$ Yao $\mathrm{Hu}^{1,2}$; Yang Song ${ }^{3}$; Min Kang ${ }^{1,2} ;$ Meng Zhang ${ }^{1,2}$; \\ Xiaoling Deng ${ }^{1,2}$; Jun Yuan ${ }^{4}$; Wenbo $\mathrm{Xu}^{3, * *}$; Baisheng $\mathrm{Li}^{1,2, *}$
}

On May 20, 2021, 75-year-old female (Case A) went to a local hospital due to pharyngeal pain and low-grade fever in Liwan District, Guangzhou City, Guangdong Province. The oropharyngeal swab from Case A tested preliminarily positive for severe acute respiratory syndrome coronavirus 2 (SARS-CoV2)/coronavirus disease 2019 (COVID-19) virus using a quantitative real-time reverse transcription polymerase chain reaction (RT-qPCR) method after a hospital visit. In the early morning of May 21, 2021, the recollected specimens were confirmed to be positive for COVID-19 for this patient with RT-qPCR tests by Guangdong Provincial CDC and Guangzhou City CDC. Subsequently, Guangdong Province began immediately taking a series of rigorous intervention measures including social distancing, community management, and lockdown to contain this outbreak. A total of 4 cases were confirmed with COVID-19 infections between May 23 and May 26 (Table 1). These patients were transported immediately to the local designated treatment hospital for isolated treatment by ambulance after COVID-19 virus infection was confirmed.
The viral ribonucleic acid (RNA) was extracted directly from $200-\mu \mathrm{L}$ swab samples with Viral RAN/DNA Mini Kit (Invitrogen, USA). Highthroughput sequencing was performed for 5 COVID-19 virus samples using Illumina (USA) and MGI Tech Co., Shenzhen, China. Nucleotide (nt) and amino acid (AA) differences between the 5 virus genome sequences from this study and the reference sequence Wuhan-Hu-1 were analyzed using the programs BioEdit and online tool "Nextclade" (https:// clades.nextstrain.org/) (1).

The 5 specimens from Case A, Case B, Case C, Case $\mathrm{D}$, and Case $\mathrm{E}$ were tentatively designated as XG5138Case A-GZ-2021-05-21, XG5370-Case B-GZ-202105-23, XG5571-Case C-MM-2021-05-24, XG5647Case D-GZ-2021-05-26, and XG5645-Case E-GZ2021-05-26, respectively, in this study. Compared with the reference genome sequence Wuhan-Hu-1 (2-3), the genome sequences coverage of these 5 strains were $99.89 \%, 99.85 \%, 99.86 \%, 99.86 \%$, and $99.87 \%$, respectively. Based on the "Pango lineages" rule, the 5 virus strains from this study were assigned to delta (B.1.617.2), which was also known as variant of

TABLE 1. Demographic characteristics of the cases and specimen testing information.

\begin{tabular}{|c|c|c|c|c|c|c|c|c|c|}
\hline \multirow{2}{*}{ Case ID } & \multirow{2}{*}{ Sample number } & \multirow[b]{2}{*}{ Gender } & \multirow{2}{*}{$\begin{array}{c}\text { Age } \\
\text { (years) }\end{array}$} & \multirow[b]{2}{*}{ Report city } & \multirow{2}{*}{$\begin{array}{c}\text { Date of first } \\
\text { positive } \\
\text { detection of } \\
\text { COVID-19 virus }\end{array}$} & \multicolumn{4}{|c|}{ Ct value (ORF1ab/N) by RT-qPCR } \\
\hline & & & & & & Specimen type & Daan & Bojie & Mingde \\
\hline \multirow{2}{*}{ Case A } & \multirow{2}{*}{$\begin{array}{c}\text { XG5138-Case } \\
\text { A-GZ-2021-05-21 }\end{array}$} & \multirow{2}{*}{ Female } & \multirow{2}{*}{75} & \multirow{2}{*}{ Guangzhou } & \multirow{2}{*}{ May 21, 2021} & Nasopharyngeal swab & $17 / 16$ & $\mathrm{~N} / \mathrm{A}$ & $18 / 21$ \\
\hline & & & & & & Oropharyngeal swab & $20 / 19$ & $\mathrm{~N} / \mathrm{A}$ & $18 / 21$ \\
\hline \multirow{2}{*}{ Case B } & \multirow{2}{*}{$\begin{array}{c}\text { XG5370-Case } \\
\text { B-GZ-2021-05-23 }\end{array}$} & \multirow{2}{*}{ Male } & \multirow{2}{*}{75} & \multirow{2}{*}{ Guangzhou } & \multirow{2}{*}{ May 23, 2021} & Nasopharyngeal swab & $21 / 22$ & N/A & $26 / 26$ \\
\hline & & & & & & Oropharyngeal swab & $21 / 21$ & N/A & $23 / 23$ \\
\hline \multirow{2}{*}{ Case C } & \multirow{2}{*}{$\begin{array}{c}\text { XG5571-Case } \\
\text { C-MM-2021-05-24 }\end{array}$} & \multirow{2}{*}{ Female } & \multirow{2}{*}{47} & \multirow{2}{*}{ Maoming } & \multirow{2}{*}{ May 25, 2021} & Nasopharyngeal swab & $16 / 13$ & $14 / 15$ & N/A \\
\hline & & & & & & Oropharyngeal swab & $20 / 18$ & $19 / 20$ & $\mathrm{~N} / \mathrm{A}$ \\
\hline Case D & $\begin{array}{c}\text { XG5647-Case } \\
\text { D-GZ-2021-05-26 }\end{array}$ & Male & 11 & Guangzhou & May 26, 2021 & Nasopharyngeal swab & $16 / 13$ & $\mathrm{~N} / \mathrm{A}$ & $16 / 15$ \\
\hline Case $\mathrm{E}$ & $\begin{array}{c}\text { XG5645-Case } \\
\text { E-GZ-2021-05-26 }\end{array}$ & Female & 73 & Guangzhou & May 26, 2021 & Nasopharyngeal swab & $19 / 17$ & $\mathrm{~N} / \mathrm{A}$ & $19 / 19$ \\
\hline
\end{tabular}

Note: N/A=not applicable.

Abbreviation: $\mathrm{RT}-\mathrm{qPCR}=$ quantitative real-time reverse transcription $\mathrm{PCR}$. 
concern (VOC) Delta (Figure 1) (4-6).

Compared with the reference genome sequence Wuhan-Hu-1, 5 strains shared 35 nucleotide variation sites (G210T, C241T, C1191T, C1267T, C3037T, C5184T, T6023C, C9598T, C9891T, T11418C, T12946C, C14408T, G15451A, C16466T, C18176T, A20262G, A21137G, C21618G, G21987A, T22917G, C22995A, A23403G, C23604G, G24410A, G25088T, C25469T, A25562G, T26767C, T27638C, C27739T, C27752T, A28461G, G28881T, G29402T, and G29742T) and 7 deletion mutations (22029-22035, 28248-28254, and 28274). Except for the mutations above, another variation site (C27092T) was observed in genome of the strain XG5571-Case C-MM-202105-24, XG5647-Case D-GZ-2021-05-26, and
XG5645-Case E-GZ-2021-05-26. XG5370-Case BGZ-2021-05-23 had a unique variation site (T21673C), but not the C27092T variation.

By comparing deduced amino acid sequences, the 5 strains displayed 29 AA variation sites (M:I82T; N:D63G, R203M, D377Y; ORF1a:P309L, P1640L, Y1920H, A3209V, V3718A; ORF1b:P314L, G662S, P1000L, P1570L, K2557R; ORF3a:S26L, Q57R; ORF7a:V82A, L116F, T120I; ORF9b:T60A; S:T19R, G142D, R158G, L452R, T478K, D614G, P681R, $\mathrm{D} 950 \mathrm{~N}$, and V1176F) and 4 deletion mutations (ORF8:D119del, F120del; S:F156del, and R157del).

The delta (B.1.617.2) was first identified in India in October 2020 and its spike protein had 9-10 characteristic mutations: T19R, (G142D), 156del, 157del, R158G, L452R, T478K, D614G, P681R, and

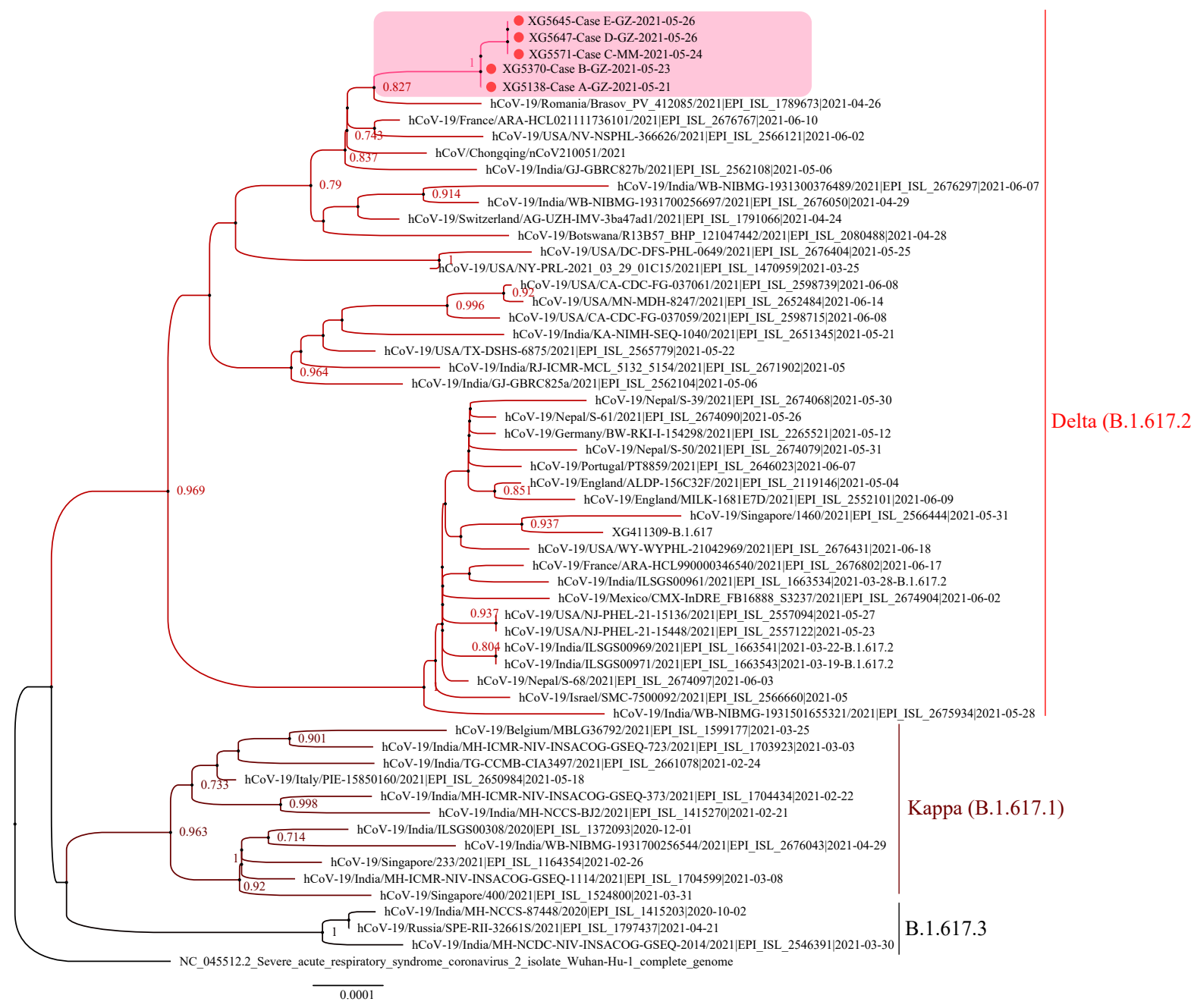

FIGURE 1. Neighbor-joining phylogenetic tree based on the whole-genome sequences of the delta (B.1.617.2) COVID-19 virus representative strains.

Note: The delta (B.1.617.2), kappa (B.1.617.1) and B.1.617.3 strains were marked on the right side. The five Guangzhou delta strains were indicated by red dots and highlighted by light red background. The tree was rooted using the Wuhan reference strain. 
D950N (4-5,7). According to the current data of the World Health Organization (WHO), the delta (B.1.617.2) spread rapidly to 74 countries, areas, or territories (December 2019-June 8, 2021) (5). The epidemiological investigation showed that Case A was the index case and Case $\mathrm{B}$ (husband) was a close contact of Case A. Furthermore, Case C and Case E were at high risk of exposure due to Case A's activities (Case $\mathrm{C}$ as a waiter served Case $\mathrm{A}$ in a restaurant, and Case $\mathrm{E}$ went to same restaurant for dinner at the same time). Lastly, Case D was the grandson of Case E and was therefore a close contact. The whole gene sequencing results showed that Case $\mathrm{C}$, Case $\mathrm{D}$, and Case $\mathrm{E}$ added a common mutation site (C27092T) based on the sequence of Case A, and Case B possessed a new mutation site (T21673C) compared with Case A's sequence.

This Guangzhou outbreak is the first local transmission caused by delta (B.1.617.2) in China. The transmissibility and pathogenicity of these mutant variants urgently need more attention.

doi: $10.46234 / \mathrm{ccdcw} 2021.151$

* Corresponding authors: Wenbo Xu, xuwb@ivdc.chinacdc.cn; Baisheng Li, libsn@126.com.

\footnotetext{
${ }^{1}$ Guangdong Provincial Center for Disease Control and Prevention, Guangzhou, Guangdong, China; ${ }^{2}$ Guangdong Workstation for Emerging Infectious Disease Control and Prevention, Chinese
}

Academy of Medical Sciences, Guangzhou, Guangdong, China; ${ }^{3}$ National Institute for Viral Disease Control and Prevention, China CDC, Beijing, China; ${ }^{4}$ Guangzhou Center for Disease Control and Prevention, Guangzhou, Guangdong, China.

\& Joint first authors.

Submitted: June 25, 2021; Accepted: June 28, 2021

\section{REFERENCES}

1. Hadfield J, Megill C, Bell SM, Huddleston J, Potter B, Callender C, et al. Nextstrain: real-time tracking of pathogen evolution. Bioinformatics 2018;34(23):4121 - 3. http://dx.doi.org/10.1093/bioinformatics/bty407.

2. Tan WJ, Zhao X, Ma XJ, Wang WL, Niu PH, Xu WB, et al. A novel coronavirus genome identified in a cluster of pneumonia cases-Wuhan China 2019-2020. China CDC Wkly 2020;2(4):61 - 2. http://dx. doi.org/10.46234/ccdcw2020.017.

3. Wu F, Zhao S, Yu B, Chen YM, Wang W, Song ZG, et al. A new coronavirus associated with human respiratory disease in China. Nature 2020;579(7798):265 - 9. http://dx.doi.org/10.1038/s41586-020-2008-3.

4. Rambaut A, Holmes EC, O'Toole Á, Hill V, McCrone JT, Ruis C, et al. A dynamic nomenclature proposal for SARS-CoV-2 lineages to assist genomic epidemiology. Nat Microbiol 2020;5(11):1403 - 7. http://dx. doi.org/10.1038/s41564-020-0770-5.

5. World Health Organization. Coronavirus disease (COVID-19) weekly epidemiological update and weekly operational update. https://www. who.int/publications/m/item/weekly-epidemiological-update-on-covid19. [2021-6-8].

6. CDC. SARS-CoV-2 variant classifications and definitions. https://www. cdc.gov/coronavirus/2019-ncov/variants/variant-info.html. [2021-6-23].

7. Ye S, Zhang YJ, Zhao X, Yu Z, Song Y, Tan ZP, et al. Emerging variants of B.1.617 lineage identified among returning Chinese employees working in India-Chongqing Municipality, China, April 2021. China CDC Wkly 2021;3(19):409-10. http://dx.doi.org/10.46234/ccdcw 2021.109. 